\title{
SARS-CoV-2: una revisión rápida de algunas de las opciones de tratamiento disponibles.
}

SARS-CoV-2: a quick review of some of the available treatment options.

https://doi.org/10.54139/salus.v25i3.131

Mariajosé Rodríguez-Núñez' (D) Mariangel Delgado² (D) Héctor R Rangel ${ }^{1}$ (D)

\section{RESUMEN}

Introducción: Con más de 244 millones de casos en todo el mundo, la pandemia de SARS-CoV-2 ha afectado a casi todos los países del planeta. El impacto en los sistemas salud, economía, educación y científicos, entre otros, ha sido significativo. La búsqueda de herramientas terapéuticas para el tratamiento y control de la nueva enfermedad, COVID-19, ha sido intensa, pero aún no ha sido posible seleccionar un fármaco específico y eficaz para el tratamiento de la misma. Metodología: el objetivo es resumir la información existente, referente a algunos de los fármacos más comunes usados en terapia contra el COVID-19, para lograrlo se usó una metodología de búsqueda en bases de datos científicas y generales (Pubmed, Google Scholar) usando palabras claves relacionadas con el tema de interés. Los resultados muestran que las drogas más comunes usadas en el tratamiento de esta enfermedad han sido evaluadas en su mayoría en ensayos clínicos. Conclusiones: a pesar de haber transcurrido casi dos años desde el primer caso de COVID-19 no se han desarrollado o reasignado fármacos efectivos y específicos para el tratamiento de la enfermedad y algunos de los que ha sido evaluados han mostrado resultados controversiales.

Palabras clave: SARS-CoV-2, COVID-19, antivirales, coronavirus.

${ }^{1}$ Laboratorio de Virología Molecular, Centro de Microbiología y Biología Celular, Instituto Venezolano de Investigaciones Científicas, Caracas-Venezuela

'Laboratorio de Biología de Virus, Centro de Microbiología y Biología Celular, Instituto Venezolano de Investigaciones Científicas, Caracas-Venezuela

Autor de correspondencia: Héctor R Rangel (iD

E-mail: hrangel2006@gmail.com

Recibido: 03-11-2021

Aprobado: 06-12-2021
ABSTRACT

Background: With more than 244 million cases worldwide, the SARSCoV-2 pandemic has affected almost every country on the planet. Its impact on the health, economy, education and scientific systems, among others, has been significant. The search for therapeutic tools for the treatment and control of this new disease, COVID-19, has been intense, but it has not yet been possible to select a specific and effective drug for its treatment. Methodology: the objective of this work is to summarize the existing information, related to some of the most common drugs used in therapy against COVID-19, to achieve this a search methodology was used in scientific and general databases (Pubmed, Google Scholar) using keywords related to the topic of interest. The results show that the most common drugs used in the treatment of this disease have mostly been evaluated in clinical trials. Conclusions: despite almost two years passed since the first case of COVID-19, effective and specific drugs have not been developed or reassigned for the treatment of the disease and some of of the already evaluated have shown controversial results.

Keywords: SARS-CoV-2, COVID-19, antivirals, coronavirus.

\section{INTRODUCCIÓN}

En noviembre 2002 en la provincia china de Guangdong se inició la epidemia de SARS, la primera nueva enfermedad del siglo 21, que se dispersó rápidamente en diferentes países asiáticos afectando alrededor de 8422 personas con 916 fallecidos, la tasa de muerte fue $11 \%$, para ese entonces (1). Más recientemente, en diciembre 2019 se hizo pública la existencia de una enfermedad que afectaba las vías respiratorias de las personas infectadas, con características similares al SARS de 2002, que posteriormente se dispersó a niveles pandémicos en poco tiempo. En la actualidad da cuenta de un poco más de 244 millones de casos y 5 millones de muertes. Desde sus inicios las acciones para controlar su dispersión han sido muy activa y múltiples grupos de investigación alrededor del mundo están trabajando en el desarrollo de medidas para controlar la enfermedad.

El surgimiento del SARS-CoV-2 y su enfermedad asociada, el COVID-19, impuso muchos cambios a nivel global afectando desde la movilidad de las personas, la economía global y el desarrollo de las investigaciones biomédicas debido a la concentración de fondos y esfuerzos en la búsqueda de herramientas para frenar la dispersión del virus, mediante el desarrollo de vacunas y drogas potenciales, así como métodos para la detección y diagnóstico. 
El virus se transmite de una persona a la otra debido al contacto de las mucosas del tracto respiratorio con gotas de saliva contentivas de partículas virales. La proteína viral conocida como espiga se une a la enzima convertidora de angiotensina 2 y es modificada en su estructura por una proteasa celular, conocida como TMPRSS2 que se encuentra ubicada en la membrana celular, el clivaje de la espiga viral por esta proteasa expone la subunidad 2 de la espiga y promueve la fusión de las membranas viral y celular, facilitando la entrada del virus a la célula (2), mediante dos mecanismos alternativos, el primero promovido por la formación de una vacuola endocítica con la posterior fusión de la membrana del virus y la vacuola y la liberación del material genético viral en el citoplasma y alternativamente puede ocurrir una fusión directa (3) no mediada por vacuolas con la liberación directa del material genético. Desde los inicios de la pandemia, la implementación de medidas de confinamiento, uso de mascarillas, distanciamiento social entre otras, han sido una de las principales medidas para evitar el aumento de los contagios.
Debido a la falta de uno o varios fármacos específicos para controlar/erradicar la infección causada por SARS-CoV-2, los pacientes han sido tratados con el fin de disminuir los efectos de la infección mediante cuidados paliativos y así tratar de reducir la mortalidad asociada a la misma.

Dado que el desarrollo de fármacos es complejo y lento, una de las estrategias que ha sido utilizada para tratar de contrarrestar los efectos de la infección por SARS-CoV-2 y su enfermedad asociada, el COVID-19, ha sido la reutilización de fármacos ya conocidos y la predicción de algunas moléculas potenciales basadas en el uso de herramientas bioinformáticas (4).

Diferentes blancos potenciales de ser inhibidos han sido detectados y algunos han mostrado ser susceptibles a la intervención farmacológica. En la figura 1 se muestra un esquema del ciclo de replicación viral de SARS-CoV-2 señalando los puntos potenciales de ser inhibidos por fármacos conocidos o nuevos.

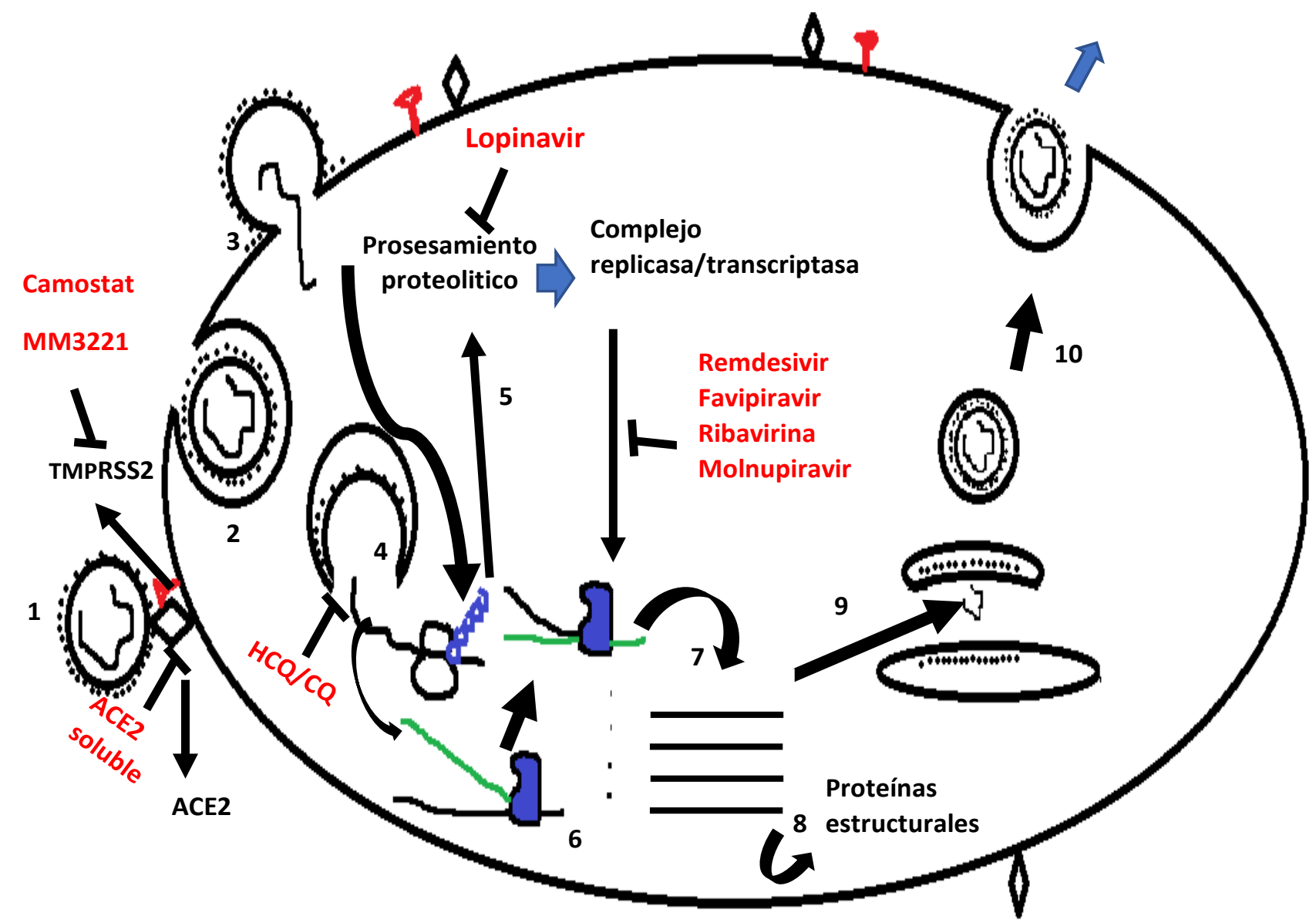

Figura 1. Ciclo replicativo de SARS-CoV-2. Se muestran algunos de los sitios estudiados como puntos potenciales de control del proceso de replicación. 1) Unión del virus al receptor ACE2 y procesamiento proteolítico de la espiga viral por parte de la proteasa TMPRSS2, 2) Formación de la vesícula endocítica, 3) Fusión directa del virus y la membrana celular liberando el material genético, 4) fusión de la membrana viral y el endosoma para liberar el material genético al citoplasma. 5) Síntesis de proteínas no estructurales y procesamiento proteolítico mediante la enzima 3CLPro. 6) Síntesis de RNA (-), 7) Replicación de RNA (+) genómico, 8) Producción de proteínas no estructurales, 9) Modificación de proteínas a nivel Golgi y ensamblaje viral, 10) exportación de partículas virales y liberación de las mismas al medio extracelular. Quintero V. 2021 
Algunos de los fármacos/compuestos más comunes que se han usado en ensayos clínicos o in vitro para inhibir la replicación de SARS-CoV-2 se enumeran a continuación.

1) Cloroquina/Hidroxicloroquina. La cloroquina (CQ) y la hidroxicloroquina ( $\mathrm{HCQ}$ ) han sido ampliamente usadas, desde hace muchos años, en el tratamiento de la malaria. Se sabe que es un fármaco seguro y bien tolerable, pero cuyo efecto positivo puede ser opacado por su toxicidad (5). Desde finales de la década de 1960 se tiene información de su actividad antiviral (6,7), más recientemente su efectividad ha sido evaluada contra coronavirus como SARS-CoV y OC43 $(8,9)$, sin embargo, su uso contra otros virus como Dengue o Zika, no mostró efectos beneficiosos $(10,11)$. Gao y col. (12) realizaron un estudio de eficacia de la $C Q$ como antiviral para el control de la infección por SARS-CoV-2, el estudio demostró una reducción en la "carga viral" en el grupo tratado con $\mathrm{CQ}$, sin embargo, debido al tamaño muestral del estudio $(\mathrm{N}=36)$ los resultados han sido criticado $(13,14)$. El mecanismo de acción de acción de la $\mathrm{CQ} / \mathrm{HCQ}$ es mediado por una alcalinización del endosoma, lo que evita la fusión de las membranas viral y endosomal, pero es importante señalar que SARS-CoV-2 podría estar usando una ruta de entrada no dependiente de endosomas que no sería afectada por la acción de la $\mathrm{CQ} / \mathrm{HCQ}$, lo que explicaría la falta de actividad terapéutica de la $\mathrm{CQ} / \mathrm{HCQ}$ en los pacientes con COVID-19.

2) Lopinavir/Ritonavir (LPV/RTV). Lopinavir y Ritonavir son fármacos usados en la terapia contra HIV-1. El blanco afectado por LPV/RTV son las proteasas virales lo que afectaría el procesamiento proteolítico de las poliproteínas sintetizadas al inicio del proceso de replicación. Estudios in vitro han demostrado la efectividad del LPV contra SARSCoV, un coronavirus relacionado con SARS-CoV-2 $(15,16)$

Los resultados de ensayos clínicos en pacientes con COVID-19 no han mostrado eficiencia en el uso de estos inhibidores, sin embargo un posible retardo en el inicio de la terapia basada en LPV/RTV podría ser responsable de escaso impacto de esta terapia en el tratamiento del COVID-19 (17)

3) Ivermectina. La ivermectina fue aprobada para su uso en humanos en 1987, como un fármaco antiparasitario y ha sido usada para el tratamiento de varios millones de personas con oncocercosis (18). El mecanismo de acción de esta droga se asocia a la inhibición del transporte nuclear de proteínas virales, evidenciado en ensayos con HIV-1 y dengue donde adicionalmente se observó una inhibición de la replicación de estos virus (19). Además, estudios in silico sugieren que la ivermectina posee una alta especifidad por la polimerasa viral de SARS-CoV-2 (20), sin embargo no hay pruebas experimentales que soporten esta observación. También se le ha asociado un efecto modulador de la tormenta de citocinas, mediante le reducción de la producción de interleucina 6 (IL-6) y factor de necrosis tumoral alfa (TNF-alfa). Un estudio realizado en pacientes con síntomas moderados mostró una reducción en el tiempo requerido para no detectar el virus mediante RT-PCR después de iniciado el tratamiento, 10 días en el grupo tratado con ivermectina versus 13 días en el grupo control (22). Un segundo estudio demostró, de manera retrospectiva, que en pacientes con condiciones moderadas a graves, el uso de la droga redujo la tasa de mortalidad del grupo tratado en comparación con el grupo control, 15 versus $25 \%$ respectivamente, dentro del mismo estudio, en aquellos pacientes graves se observó una reducción de la mortalidad aún más marcada en aquellos que recibieron ivermectina, $38 \%$ versus $80 \%$ (22).

4) Remdesivir. El remdesivir es un inhibidor de RNA polimerasas. Se ha descrito su actividad antiviral contra virus como Ebola, MERS, Nipah SARS-CoV, entre otros (23), por su parte el remdesivir parece mediar su efecto como un terminador de cadena, sin embargo aún no está claro su mecanismo de acción.

En un estudio llevado a cabo por Beigel y col (24), se muestra una reducción significativa en el tiempo de hospitalización en pacientes que recibieron remdesivir intravenoso en comparación al grupo control, sin embargo en un estudio de "interim WHO solidarity clinical trial" (25), indica que el remdesivir, entre otras drogas, posee poca o nula efectividad contra el COVID-19 en relación a la tasa de mortalidad, tiempo de hospitalización, etc. (25).

5) Favipiravir/Molnupiravir/Ribavirina. Favipiravir y Molnupiravir afectan la actividad de la RNA polimerasa viral. Favipiravir ha mostrado actividad contra virus como influenza, el virus del oeste del Nilo (WNV), fiebre amarilla, bunyavirus, entre otros (26), por ser un análogo de guanidina, la inhibición de la RNA polimerasa conduce a un aumento en la tasa de mutaciones y por ende a un incremento en la cantidad de virus no viables (mutagénesis letal) (27). Un estudio llevado a cabo para determinar la efectividad del Favipiravir en pacientes con COVID-19 sugiere que el uso de este medicamento se traduce en una reducción del tiempo de hospitalización (28); sin embargo, el estudio no fue concluyente basado en la estadística.

Por su parte, el Molnupiravir, es incorporado en el ARN e induce la unión de nucleótidos errados en el mismo, lo que conduce a un aumento en la tasa de mutación viral más allá del nivel biológicamente sostenible para que el virus mantenga su capacidad replicativa (29).

Por su parte la ribavirina, es un antiviral de amplio espectro usado en el tratamiento de enfermedades causadas por virus como influenza, hantavirus, HIV-1, entre otros. El mecanismo de acción no se conoce de manera precisa; sin embargo, es conocido que inhibe la inosin monofosfato deshidrogenasa (IMPDH), lo que conduce a una reducción en la concentración de GTP que afecta la síntesis de RNA. Algunos estudios sugieren que puede promover la 
mutagénesis letal en virus como HCV $(30,31)$. En el caso de COVID-19, se ha sugerido su uso de manera combinada con interferón y LPV/RTV, los resultados de estos ensayos sugieren un beneficio de la combinación en cuanto al tiempo de hospitalización (32)

6) MM3122. El MM3122 es un nuevo tipo de inhibidor de la enzima TMPRSS2, una proteasa de membrana que media el corte proteolítico entre las subunidades $\mathrm{S} 1$ y $\mathrm{S} 2$ de la espiga viral de SARS-CoV-2, favoreciendo así el proceso de fusión de membranas. El MM3122 presenta mejor actividad inhibitoria contra TMPRSS2 que otros inhibidores conocidos como el Camostat. Estudios in silico, acompañados de ensayos in vitro demuestran una fuerte inhibición de la TMPRSS2 y en consecuencia la inhibición de la replicación de SARS-CoV-2 in vitro (2); sin embargo, los estudios de fase I, II y III son necesarios antes de poder indicar que este compuesto es apropiado para su uso como agente terapéutico en el tratamiento del COVID-19.

\section{CONCLUSIONES}

Aun después de casi dos años de haberse declarado el primer caso de SARS-CoV-2 en China, que ha afectado negativamente a toda la humanidad, no se dispone de un fármaco especifico con alta potencialidad antiviral para el tratamiento del COVID-19. Diversos grupos de investigación a nivel mundial llevan a cabo grandes esfuerzos en la búsqueda de nuevas herramientas terapéuticas para el tratamiento de esta nueva enfermedad. El desarrollo de vacunas ha surgido como una herramienta eficiente en el control de la enfermedad, pero ha sido parcialmente opacado por el impacto de las diversas variantes del virus que se ha seleccionado en numerosas regiones del planeta. Estos eventos dejan claro la necesidad del desarrollo de una terapia que pueda coadyuvar con los procesos de vacunación en control de la dispersión viral. Varias drogas como remdesivir, ivermectina hidroxicloroquina y Lopinavir se han incluido en los esquemas de tratamiento de pacientes; sin embargo, los resultados obtenidos en los diferentes estudios no son del todo concluyentes y se han generado discrepancias entre estos estudios debido a la falta controles apropiados, así como a la heterogeneidad de los grupos de poblaciones de cada estudio; a pesar de ello, se siguen usando en diversos países, incluido el nuestro. Nuevas drogas como es el caso de Molnupiravir y MM3221, introducen nuevas herramientas en los estudios farmacológicos y se convierten en compuestos líderes para el desarrollo de nuevas moléculas.

El tratamiento combinado para la erradicación del SARS-CoV-2 debe ser la meta, por encima de cualquier monoterapia, de manera de disminuir la probabilidad de seleccionar variantes resistentes a la quimioterapia, que eventualmente compliquen el panorama al sumarse a las variantes de escape. Nuevos y múltiples estudios son necesarios para poder determinar la efectividad de los fármacos reasignados o nuevos para el tratamiento del COVID-19.

\section{REFERENCIAS BIBLIOGRÁFICAS}

1. Chan KS, Zheng JP, Mok YW, Li YM, Liu YN, Chu CM, Ip MS. SARS: prognosis, outcome and sequelae. Respirology. 2003;8 Suppl(Suppl 1):S36-S40. https://doi.org/10.1046/j.14401843.2003.00522.x

2. Mahoney M, Damalanka VC, Tartell MA, Chung DH, Lourenço $A L$, Pwee D, et al. A novel class of TMPRSS2 inhibitors potently block SARS-CoV-2 and MERS-CoV viral entry and protect human epithelial lung cells. Proc Natl Acad Sci U S A. 2021;118(43):e2108728118. https://doi.org/10.1073/ pnas. 2108728118 .

3. Haixia S, Yechun X, Hualiang J. Drug discovery and development targeting the life cycle of SARS-CoV-2, Fundamental Research. 2021; 1(2):151-165 https://doi.org/10.1016/j.fmre.2021.01.013.

4. Ortega JT, Zambrano JL, Jastrzebska B, Liprandi F, Rangel $\mathrm{HR}$, Pujol FH. Understanding severe acute respiratory syndrome coronavirus 2 replication to design efficient drug combination therapies. Intervirology. 2020;63(1-6):2-9. https:// doi.org/10.1159/000512141.

5. Frisk-Holmberg $\mathrm{M}$, Bergqvist $\mathrm{Y}$, Englund $\mathrm{U}$. Chloroquine intoxication. Br J Clin Pharmacol. 1983;15(4):502-503. https:// doi.org/10.1111/j.1365-2125.1983.tb01540.x

6. Inglot $A D$. Comparison of the antiviral activity in vitro of some non-steroidal anti-inflammatory drugs. J Gen Virol. 1969;4(2):203-14. https://doi.org/10.1099/0022-1317-4-2-203.

7. Miller DK, Lenard J. Antihistaminics, local anesthetics, and other amines as antiviral agents. Proc Natl Acad Sci U S A. 1981;78(6):3605-3609. https://doi.org/10.1073/pnas.78.6.3605

8. Keyaerts E., Vijgen L., Maes P., Neyts J., Ranst M.V. In vitro inhibition of severe acute respiratory syndrome coronavirus by chloroquine. Biochem. Biophys. Res. Commun. 2004;323:264268. https://doi.org/10.1016/j.bbrc.2004.08.085

9. Keyaerts E., Li S., Vijgen L., Rysman E., Verbeeck J., Van Ranst M. et al. Antiviral activity of chloroquine against human coronavirus OC43 infection in newborn mice. Antimicrob. Agents Chemother. 2009;53:3416-3421. https://doi.org/10.1128/ AAC.01509-08.

10. Tricou V, Minh NN, Van TP, Lee SJ, Farrar J, Wills B, et al. A randomized controlled trial of chloroquine for the treatment of dengue in Vietnamese adults. PLoS Neglected Trop. Dis. 2010;4:e785. https://doi.org/10.1371/journal.pntd.0000785

11. Maheshwari R.K., Srikantan V., Bhartiya D. Chloroquine enhances replication of Semliki Forest virus and encephalomyocarditis virus in mice. J. Virol. 1991; 65:992-995

12. Gao J, Tian Z, Yang X. Breakthrough: chloroquine phosphate has shown apparent efficacy in treatment of COVID-19 associated pneumonia in clinical studies. Biosci Trends. 2020;14(1):72-73

13. Megarbane B. Chloroquine and hydroxychloroquine to treat COVID-19: between hope and caution. Clin Toxicol (Phila). 2021;59(1):70-71. https://doi.org/10.1080/15563650.2020.174 8194. 
14. Carafoli E. Chloroquine and hydroxychloroquine in the prophylaxis and therapy of COVID-19 infection. Biochem Biophys Res Commun. 2021; 538:156-162. https://doi. org/10.1016/j.bbrc.2020.09.128

15. Chen F, Chan KH, Jiang Y, Kao RY, Lu HT, Fan KW et al. In vitro susceptibility of 10 clinical isolates of SARS coronavirus to selected antiviral compounds. J Clin Virol. 2004;31(1):69-75.

16. Chu CM, Cheng VC, Hung IF, Wong MM, Chan KH, Chan KS et al. Role of lopinavir/ritonavir in the treatment of SARS: initial virological and clinical findings. Thorax. 2004;59(3):252-256.

17. Cao B., Wang Y., Wen D., Liu W., Wang J., Fan G., et al. A trial of lopinavir-ritonavir in adults hospitalized with severe Covid-19. N Engl J Med. 2020;382(19):1787-1799. https://doi. org/10.1056/NEJMoa2001282.

18. Wehbe Z, Wehbe M, Iratni R, Pintus G, Zaraket H, Yassine HM, et al. Repurposing ivermectin for COVID-19: Molecular aspects and therapeutic possibilities. Front Immunol. 2021;12:663586. https://doi.org/10.3389/fimmu.2021.663586

19. Wagstaff KM, Sivakumaran $H$, Heaton SM, Harrich D, Jans DA. Ivermectin is a specific inhibitor of importin $\alpha / \beta$-mediated nuclear import able to inhibit replication of HIV-1 and dengue virus. Biochem J 2012; 443(3):851-856. 10.1042/BJ20120150

20. Gupta PSS, Biswal S, Panda SK, Ray AK, Rana MK. Binding mechanism and structural insights into the identified protein target of COVID-19 and importin- $\alpha$ with in-vitro effective drug ivermectin. J Biomol Struct Dyn 2020; 1-10. https://doi.org/10.1 080/07391102.2020.1839564

21. Ahmed S, Karim MM, Ross AG, Hossain MS, Clemens JD, Sumiya MK, et al. A five-day course of ivermectin for the treatment of COVID-19 may reduce the duration of illness. Int J Infect Dis 2020;103:214-216. https://doi.org/10.1016/j. ijid.2020.11.191

22. Rajter JC, Sherman MS, Fatteh N, Vogel F, Sacks J, Rajter $\mathrm{JJ}$. Use of ivermectin is associated with lower mortality in hospitalized patients with coronavirus disease 2019: The ivermectin in COVID nineteen study. Chest 2021;159(1):85-92. https://doi.org/10.1016/j.chest.2020.10.009

23. Rommasi F, Nasiri MJ, Mirsaiedi M. Antiviral drugs proposed for COVID-19: action mechanism and pharmacological data. Eur Rev Med Pharmacol Sci. 2021;25(11):4163-4173. https://doi. org/10.26355/eurrev_202106_26060
24. Beigel JH, Tomashek KM, Dodd LE, Mehta AK, Zingman BS, Kalil AC, et al. ACTT-1 Study group members. Remdesivir for the treatment of Covid-19 - Final Report. N Engl J Med. 2020;383(19):1813-1826. https://doi.org/10.1056/ NEJMoa2007764

25. WHO Solidarity Trial Consortium Repurposed antiviral drugs for Covid-19-interim WHO solidarity trial results. N. Engl. J. Med. 2021;384, 497-511.

26. Agrawal U, Raju R, Udwadia ZF. Favipiravir: a new and emerging antiviral option in COVID-19. Med J Armed Forces India 2020; 76: 370-376.

27. Mishra SK, Tripathi T. One year update on the COVID-19 pandemic: Where are we now? Acta Trop. 2021;214:105778. https://doi.org/10.1016/j.actatropica.2020.105778

28. Udwadia ZF, Singh P, Barkate H, Patil S, Rangwala S, Pendse A, et al. Efficacy and safety of favipiravir, an oral RNA-dependent RNA polymerase inhibitor, in mild-to-moderate COVID-19: A randomized, comparative, open-label, multicenter, phase 3 clinical trial. Int J Infect Dis. 2021;103:62-71. https://doi. org/10.1016/j.jijid.2020.11.142.

29. Kabinger F, Stiller C, Schmitzová J, Dienemann C, Kokic G, Hillen HS, et al. Mechanism of molnupiravir-induced SARSCoV-2 mutagenesis. Nat Struct Mol Biol. 2021;28(9):740-746. https://doi.org/10.1038/s41594-021-00651-0.

30. Crotty S, Cameron C, Andino R. Ribavirin's antiviral mechanism of action: lethal mutagenesis? J. Mol. Med. 2002;80(2):86-95.

31. Thomas E., Ghany M.G., Liang T.J. Chemotherapy, The application and mechanism of action of ribavirin in therapy of hepatitis C. J. Antiviral Chem. 2012;23(1):1-12

32. Hung IF, Lung KC, Tso EY, Liu R, Chung TW, Chu MY, et al. Triple combination of interferon beta- $1 \mathrm{~b}$, lopinavir-ritonavir, and ribavirin in the treatment of patients admitted to hospital with COVID-19: an open-label, ramdomised, phase 2 trial. Lancet. 2020;395(10238):1695-1704. doi: 10.1016/S01406736(20)31042-4 